Mots. Les langages du politique

Guerres et paix. Débats, combats, polémiques

\title{
Michel Bréal, le marathon, l'olympisme et la paix
}

\section{Marc Décimo et Pierre Fiala}

\section{OpenEdition}

Journals

Édition électronique

URL : https://journals.openedition.org/mots/3743

DOI : $10.4000 /$ mots. 3743

ISSN : 1960-6001

\section{Éditeur}

ENS Éditions

\section{Édition imprimée}

Date de publication : 1 novembre 2004

Pagination : 127-135

ISBN : 2-84788-064-X

ISSN : 0243-6450

\section{Référence électronique}

Marc Décimo et Pierre Fiala, « Michel Bréal, le marathon, l'olympisme et la paix », Mots. Les langages du politique [En ligne], 76 | 2004, mis en ligne le 21 avril 2008, consulté le 23 avril 2022. URL : http:// journals.openedition.org/mots/3743; DOI : https://doi.org/10.4000/mots.3743 
Marc DÉCIMO $^{1}$, Pierre FIALA ${ }^{2}$

\section{Des mots en politique}

\section{Michel Bréal, le marathon, l'olympisme et la paix}

Helléniste de renom, traducteur de la Grammaire comparée de Franz Bopp, fondateur avec Gaston Paris et Gabriel Monod de l'École pratique des hautes études, où il invita Ferdinand de Saussure, secrétaire de la Société de linguistique de Paris jusqu'à sa mort, Michel Bréal (1832-1915), esprit libre et imaginatif, est l'inventeur de la sémantique. Et du marathon ${ }^{3}$, comme chacun sait.

De 1864 à 1905, le savant éminent qu'il était enseigna au Collège de France, où il fut le prédécesseur d'Antoine Meillet, une conception systématique et néanmoins concrète de l'histoire des langues, qu'il proposa ${ }^{4}$ de nommer sémantique. Il ouvrait par là un champ de connaissance linguistique traitant de l'évolution générale du sens des mots, évitant d'une part le formalisme abstrait des néogrammairiens et d'autre part l'anecdotisme romanesque qui présidait alors à l'histoire romantique des mots. Le citoyen engagé qu'il fut défendit activement plusieurs réformes de l'éducation. Il fut attaché toute sa vie à l'idée de civisme républicain et manifesta son zèle pour le bien public sous de nombreuses formes : patriotisme et philanthropie, amour du progrès et souci de la justice, dévouement aux belles idées abstraites. Selon lui, les conditions de possibilité pour vivre en paix ne se réaliseraient que par l'instauration d'un État de droit et l'exercice de la paix civile; de même, la paix internationale ne serait possible qu'en défendant la laïcité, les vertus de la science et des arts, et le sport. Ainsi Bréal défendit-il la poésie en soulignant combien l'engouement pour Mistral et les félibres était partagé par les lettrés allemands et français ${ }^{5}$. Ainsi soutint-il

1. Université d'Orléans - marcdecimo@aol.com

2. Université Paris 12, Val de Marne-CEDITEC - fiala@univ-paris12.fr

3. Peut-être sa naissance à Landau, ville de Bavière qui donna son nom à un type fameux de voiture, le prédestinait-elle facétieusement à la création métonymique.

4. Essai de sémantique, Paris, Hachette, 1897.

5. M. Décimo, 2000, «Quand Michel Bréal, d'origine juive et berlinoise, alsacien, félibre et citoyen écrivait à Frédéric Mistral», Revue des langues romanes, t. 104, n 1, p. 187-218. 
avec constance la naissance de Jeux olympiques et proposa-til à Pierre de Coubertin (1863-1937), pour les premières olympiades, l'équivalent de jeux floraux. Mais surtout, il eut l'idée d'y organiser le premier marathon.

Il rejoignit d'ailleurs le Comité olympique de 1894 pour organiser des jeux sportifs et suggéra alors à Coubertin d'organiser une «course de Marathon au Pnyx» (voir Annexe : Lettre 1), qui aurait une «saveur antique», offrant même une coupe d'argent à qui renouvellerait «s'il ne mourait pas» l'exploit du soldat légendaire venu apporter en 490 avant J.-C. la nouvelle de la victoire du général Miltiade sur les envahisseurs. La course fut réalisée dès les premiers Jeux de 1896, durant lesquels 19 concurrents disputèrent une course de $40 \mathrm{~km}^{6}$, remportée par le berger grec Spiridon Louis, héros d'une véritable épopée 7 . Neuf ans après, Bréal renouvela son offre de décerner personnellement une coupe en argent au vainqueur (Lettre 4). Durant toutes ces années, il ne cessa de s'intéresser (Lettre 2) à l'olympisme ${ }^{8}$ naissant, espérant vainement pouvoir l'associer à la promotion de l'art et de la poésie sur le modèle antique (Lettres 4 et 5). Il s'intéressa également au développement de la gymnastique (Lettres 3 et 7) manifestant des préoccupations morales (Lettre 7) et des positions pacifistes. Il désapprouva pour cette raison l'idée d'une compétition inspirée du combat des Horaces et des Curiaces et suggéra en remplacement l'organisation de concours de poésie lyrique (Lettre 5).

On ne s'étonnera pas de voir Bréal participer aussi à la Société pour l'arbitrage entre les Nations créée par Frédéric Passy (1822-1912), le père du phonéticien Paul Passy, proposer en 1913, avec une clairvoyance courageuse, un statut de neutralité pour l'Alsace-Lorraine afin d'éviter la guerre" ${ }^{9}$, ou lancer en novembre 1914 avec les professeurs de l'École pratique des hautes études un

6. Distance qui fut portée à 42,195 km aux Jeux de 1908 à Londres, pour permettre de relier le château de Windsor et le palais de Buckingham, et complaire ainsi, semble-til, à la famille royale britannique.

7. Cette première édition fut l'occasion d'une confrontation aux allures dramatiques entre le simple berger (grec) et les meilleurs spécialistes du fond des nations occidentales, qui furent distancés par celui-ci qui courut dans un temps remarquable de moins de trois heures, et fut accueilli au grand stade panathénaïque au milieu des débordements patriotiques des spectateurs, notamment les fils du souverain grec. Le berger valeureux devint, malgré lui, un héros national et prêta même son nom à une expression idiomatique signifiant «se dépasser, réussir un exploit sans précédent» (voir Charlie Lovett, The Olympic Marathon, New York, Hardcover, 1997).

8. Le terme est attesté en 1934 par le Trésor de la langue française (TLF) qui le définit comme 1'«Ensemble des activités relatives aux Jeux olympiques, des statuts réglementant l'organisation et le déroulement des Jeux».

9. M. Bréal, 1913, «La neutralisation de l'Alsace-Lorraine», La paix par le droit, 25 janvier, p. 36 . 
«Appel aux pays neutres» dans le Journal de Genève ${ }^{10}$. Si l'engagement pacifiste du savant n'empêcha pas la guerre, sa proposition d'une «course de Marathon au Pnyx » connut un succès universel durable que les langues attestent.

\section{Une métonymie classique et une métaphore chargée de connotations}

Métonymie du lieu, plus précisément de l'évènement, le nom commun marathon n'est pas attesté comme tel dans la correspondance de Bréal à Coubertin. On trouve encore en 1895 dans le Panorama illustré des Jeux olympiques avec le programme des jeux olympiques de 1896 de Goerges Spyridis, "Course à pied, dite de Marathon» (Athènes, Éditions Wilberg, p. 77), mais l'on peut supposer que dès cette époque les commentaires et les reportages sportifs commencèrent à utiliser le terme substantifié seul, allégé de sa première partie nominale «course de...» sur le modèle des désignations plus techniques des autres épreuves : le (lancer du) javelot, le disque, le 800 mètres, etc. Cette métonymisation fut stabilisée rapidement ${ }^{11}$, tout comme son dérivé régulier, marathonien, substantif et adjectif masculin ${ }^{12}$ attesté dès 1896 selon le Dictionnaire historique de langue française d'Alain Rey ${ }^{13}$, ce qui indique le succès remporté dès 1896 par l'épreuve du marathon et son retentissement universel.

Avec sa référence particulièrement riche ${ }^{14}$, le nom de l'épreuve trouva rapidement une place à part dans les descriptifs et dans l'imaginaire des Jeux, où il évoque une sorte de limite extrême de l'effort humain et constitue le stéréotype $^{15}$ de l'épreuve olympique la plus marquée historiquement, culturellement, dramatiquement mais aussi socialement ${ }^{16}$. Autre indice de la reconnaissance acquise par le marathon: sa métaphorisation et son emploi figuré fréquent dans

10. M. Bréal et al., 1914, «Appel aux pays neutres», Journal de Genève, 16 novembre, p. 1.

11. Le TLF le signale dans le Larousse universel de 1923.

12. Le féminin, plus tardif, bien attesté aujourd'hui, n'est pas mentionné dans le $T L F$, qui mentionne en revanche le composé semi-marathon.

13. Le TLF mentionne la première attestation en 1925 «coureur de marathon» (J. Prévost, 1925, Plaisir des sports, Paris, Gallimard, p. 180).

14. Le toponyme lui-même est d'origine métonymique : «il tiroit son nom de Marathon, petit-fils d'Aloeus, qui selon la fable, avoit le soleil pour pere. Étant arrivé dans la partie maritime de l'Attique, il fonda la bourgade de Marathon, \& lui donna son nom » (article Marathon, Encyclopédie de Diderot et d'Alembert).

15. Au sens de Putnam, c'est-à-dire non pas le meilleur exemplaire (le prototype), mais l'ensemble représentatif le plus large de traits hétérogènes stabilisés.

16. Seule épreuve reposant sur un fond historique et mythique, elle a traditionnellement lieu le dernier jour des jeux. Épreuve sur route (comme les épreuves de marche) et non sur 
des tournures attributives ou appositives, sur le schéma Nom + Nom, notamment dans le langage politico-social, pour caractériser «la longueur épuisante» d'une manifestation, d'une réunion, d'une négociation. Le TLF mentionne une première attestation en 1943 avec les exemples suivants :

Wahid organise une soirée costumée orientale. Nous jouons. $\mathrm{M}^{\text {lle }}$ Debar m'étonne dans le rôle du Sphinx. C'est un marathon. Elle s'y crève (J. Cocteau, 1949, Maalesh, Paris, Gallimard, p. 86).

L'accord a été finalement conclu dans le décor habituel des marathons diplomatiques bruxellois (P. Fabra, 1966, Le Monde, 12 mai).

En apposition ou comme $2^{\mathrm{e}}$ élément de substantif composé : M. John V. Lindsay, maire de New York, a déclaré que l'accord intervenu, après une séance-marathon de trente-neuf heures, est «équitable et raisonnable» (Reuter, 1968, Le Monde, 3 janvier, p. 5, col. 6). Le voyage marathon se poursuit à un rythme hallucinant. De Gaulle [...] semble n'offrir aucune prise à la fatigue (Y. Courrière, 1971, Les feux du désespoir, Paris, Fayard, p. 184).

Outre les traits indiquant la longueur, la tension, la fatigue, marquant souvent aussi la volonté et la résistance des acteurs eux-mêmes, la connotation comporte comme autres traits la présence d'un objectif à atteindre, lequel est d'ailleurs généralement atteint avec succès, grâce à la ténacité des participants. La lexicalisation de ce terme et ses usages en dehors du domaine sportif constitue l'un des multiples exemples de l'extension ${ }^{17}$ considérable du vocabulaire olympique sur le langage général, et notamment le langage politico-social médiatique, qu'il est sensé embellir, moraliser, ennoblir. Ces usages prévalent encore actuellement $^{18}$.

piste, elle va à la rencontre d'un large public en parcourant les artères des cités organisatrices. C'est à son propos que Coubertin prononça à Londres en 1898 son «l'important n'est pas de gagner mais de participer». Elle connut des affrontements dramatiques, des victoires tragiques, comme celle d'un Italien en 1908, qu'on traina jusqu'à la ligne d'arrivée, à bout de force, et qui fut déclassé pour cette raison au profit d'un Américain. L'immense succès populaire dans la seconde moitié du $20^{\mathrm{e}}$ siècle, lié à des noms devenus héroïques (Zatopek, Mimoun, Bikila, etc.) est l'une des causes du développement de la pratique du jogging, et de l'organisation par toutes les capitales mondiales de vastes rassemblements, largement sponsorisés, et orchestrés politiquement, où se mêlent sportifs professionnels et amateurs de toutes catégories et de tous âges.

17. P. Gilbert, 1974, «Les emprunts aux vocabulaires des sports», Le français dans le monde, $\mathrm{n}^{\circ} 107$, p. 54-56.

18. Le quotidien Libération utilise le terme marathon 120 fois durant l'année 2003, dont environ $3 \%$ comme toponyme (la ville, la plaine de Marathon), $15 \%$ comme substantif métonymique (le marathon de New York, de Cologne), 30 \% comme métaphore dans le domaine sportif ou du spectacle (le marathon de la danse, de natation, de la vodka, etc.), $30 \%$ comme métaphore dans le discours politico-social (dont négociation marathon, $4 \%$; réunion marathon, $4 \%$; marathon judiciaire ou juridique, $6 \%$ ). 
Ce tableau métaphorique convient à l'idéal olympique tel qu'il continue à se décliner à longueur de colonnes médiatiques, au premier ou au second degré, suivant les orientations politiques ou les humeurs des journalistes, mais il ne saurait masquer d'autres connotations plus contradictoires, voire plus violentes, qui émergèrent progressivement durant le $20^{\mathrm{e}}$ siècle. Sur plus d'un point, la naissance et l'histoire du marathon - mot et chose - illustrent les contradictions profondes de l'olympisme. Religion de la modernité, le mouvement olympique devait selon ses promoteurs transcender les haines et les antagonismes accumulés durant le $19^{\mathrm{e}}$ siècle par les guerres nationales, les guerres coloniales, les inégalités économiques et sociales, les dominations militaires, idéologiques, culturelles, religieuses en réconciliant les individus et les nations dans l'effort, le dépassement de soi et la compétition désintéressée. Contradictions sémantiques dans un ordre social désorganisé par la concurrence illimitée et la loi du plus fort, et même si les victoires, célébrées universellement, de l'ouvrier Zatopek, de l'immigré Mimoun, des bergers éthiopiens aux pieds nus, ou du facteur marocain peuvent donner l'illusion que le faible peut triompher quand les chances sont égales.

Dans l'exaltation de la compétition ${ }^{19}$ mythique, sous le symbole des valeurs de l'antiquité gréco-latines, mais aussi de la victoire militaire des Athéniens sur les armées barbares ${ }^{20}$, le marathon, après avoir été livré aux boycotts de la guerre froide, aux attaques terroristes, finit en pur spectacle, en immense cohue sur les ponts new-yorkais, en devanture médiatique et touristique livrée aux appétits sans bornes des multinationales de l'alimentation et des boissons revitalisantes. Au courage de Michel Bréal, qui voulait résister à la tentation de la guerre par la vertu des Jeux, répond aujourd'hui celui d'Albert Jacquard proclamant : «Halte aux Jeux ! ${ }^{21}$. Il est vrai que l'on est en droit de refuser, avec lui, de voir l'olympisme transformé en nouveau temple des valeurs commerciales, prônant les prétendues vertus tant individualistes que nationalistes, en outil des expériences biologiques de dopage généralisé, en objet de pur spectacle et de déchainement médiatique chauvin, et finalement en champ clos d'affrontement des cultures terroristes et sécuritaires.

19. Le terme appartient au vocabulaire économique avant d'être au centre de la terminologie sportive.

20. Le marathonien est porteur d'un message non de paix mais de victoire militaire de l'habileté et de la culture grecque, incarnées par la stratégie de Miltiade, sur la démesure et la confusion, incarnées par l'immense armée perse.

21. A. Jacquard, 2004, Halte aux Jeux ! Paris, Stock. 


\section{Annexe}

Sept lettres de Michel Bréal à Pierre de Coubertin 22 (1894-1908)

\section{Lettre 1}

Cher Monsieur,

Glion (Vaud) 15 septembre 1894

Votre aimable lettre après avoir quelque temps couru après moi, vient enfin de me rejoindre ici, et j'ai hâte d'y répondre.

Il va sans dire que je vous donne mon adhésion pleine et entière pour le Comité.

Quant à la conférence, permettez que je m'excuse pour plusieurs raisons, dont je vous donnerai une seule, que vous apprécierez certainement.

"Les Jeux Olympiques et la Paix» forment un très beau sujet, sur lequel il $\mathrm{y}$ a des choses vraies et utiles à dire. Mais il me semble qu'elles seront mieux dites par un étranger (Italien, Suisse, ou Belge) que par un Français. Inutile de vous développer cela. Il me semble que vous devriez vous adresser à un homme comme M. Bonghi ou comme M. Rod.

Si vous teniez cependant à ce que ce fût un Français, l'homme indiqué serait Jules Simon.

Ne croyez pas que je me défile. J'agis au point de vue et dans l'intérêt de votre œuvre, dont je désire la réussite.

J'ai reçu en Bretagne, où j'étais il y a trois semaines, le premier mémoire du Bulletin, et je l'ai fait circuler.

Vous faites bien de mettre en mouvement vos amis américains. Ils ont la pratique des grandes réunions et ils vous seront des auxiliaires précieux.

Puisque vous allez à Athènes, voyez donc si l'on ne peut organiser une course de Marathon au Pnyx. Cela aura une saveur antique. Si nous savions le temps qu'a mis le guerrier grec, nous pourrions établir le record. Je réclamerais pour ma part l'honneur d'offrir «la Coupe de Marathon».

Pardon pour ces lignes écrites au galop dans une chambre d'auberge. Je vous envie de pouvoir dater votre lettre du lieu où vous êtes. Avec quel plaisir je signerais

22. Ces lettres proviennent de la correspondance manuscrite de Pierre de Coubertin. Fondation du Comité international olympique, Musée olympique, Lausanne, texte établi par Marc Décimo. 
Michel Bréal

à la Bréanti

Cela satisfait nos instincts philologues.

Je ne sais si l'on vous a dit que j'ai eu la maladresse d'aller vous voir le lendemain de votre départ de Paris. Ce sera pour cet hiver.

Votre dévoué.

M.B.

\section{Lettre 2}

Cher Monsieur,

Oui, certainement, je serai à vos côtés le jour de cette conférence.

C'est très bien à vous de prendre en mains la cause de la Grèce.

Je trouve même que c'est zélé de votre part, car les bons Grecs ne me paraissent pas avoir compris, l'été dernier, tout ce qu'ils vous doivent. Mais ce sont peccadilles qu'il faut pardonner.

Affectueusement,

Michel Bréal.

Lettre 3

Cher Monsieur,

5 juillet 1897

Vous êtes bien aimable de m'inviter au Congrès du Havre. Mais que pourrais-je dire à vos gymnastes que vous ne leur disiez aussi bien que moi ? Tout au plus pourrais-je leur recommander de ne jamais négliger un épanchement de synovie au genou, car on s'en ressent le reste de ses jours, et cela rend tout déplacement difficile.

Vous continuez votre œuvre et vous faites bien. Vous faites plus pour les générations nouvelles que tous les traités de pédagogie. Je donnerais bien les palmes académiques de mon habit pour être parmi les coureurs ou lutteurs que vous allez couronner.

Affectueusement à vous.

Michel Bréal. 


\section{Lettre 4}

Cher Monsieur,

J'ai différé à dessein une réponse à votre aimable lettre, ne sachant sur quel bord de la Méditerranée il fallait la diriger. Je suppose que maintenant vous êtes de retour dans notre vieux Paris et je veux d'abord vous remercier de ne m'avoir pas oublié après tant d'années si remplies. Votre idée de choisir Rome est excel-

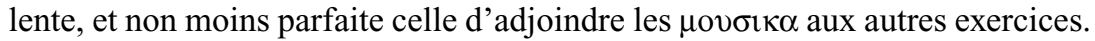
Dans un prochain $n^{\circ}$ de la Revue de Paris, vous pourrez lire mon article où l'on démontre tout ce que la Grèce des anciens temps devait aux Jeux Olympiques ${ }^{23}$. L'Iliade elle-même en a tiré son origine. C'est un assez glorieux passé et qui promet un bel avenir.

S'il y a place pour une nouvelle course de Marathon, je renouvellerai bien volontiers mon hommage d'il y a dix ans.

Peut-être cela me donnera-til des jambes pour aller vous trouver et vous applaudir.

Bien affectueusement,

Michel Bréal

87 Bd St. Michel

\section{Lettre 5}

Cher Monsieur,

J'ai eu beau y penser, les Horaces et les Curiaces ne me séduisent pas. Un match où sur six personnes engagées cinq restent sur le carreau, pour une fête pacifique et internationale, cela n'est pas très engageant.

Je leur trouve en outre un air pseudo-classique qui sent son école de David.

Il vous faut quelque chose de nouveau.

Puisque vous avez l'heureuse idée de joindre des joutes intellectuelles à vos luttes athlétiques, pourquoi ne fonderait-on pas un prix pour la meilleure pièce de vers en l'honneur des modernes $\mathrm{O} \lambda \iota \mu \pi \mathrm{\alpha} \alpha \alpha$.

Vous ferez renaître la poésie lyrique !

Seulement cela exige une mise de fonds qu'un professeur au Collège de France n'est pas en état de fournir. Il faudrait s'adresser aux poètes fortunés comme la comtesse de Noailles ou Gregh.

23. «L'Iliade d'Homère. Ses origines», Revue de Paris, 15 juin 1905, p. 712-728. En 1906, Bréal fait paraitre Pour mieux connaître Homère chez Hachette. 
Bien entendu, et ce serait l'originalité du concours, on admettrait le choix de la langue : français, anglais, allemand, italien, grec moderne.

Voilà mon idée. Je vous la livre, certain que si vous l'adoptiez, vous la ferez réussir.

Bien sympathiquement,

Michel Bréal.

\section{Lettre 6}

16 mai 1907

Cher Monsieur,

J'adhère bien volontiers à la nouvelle Société, mais je suis obligé de vous prier de vous contenter d'une adhésion morale. Le nombre des Associations dont je fais partie est vraiment excessif et prend une part disproportionnée dans mon budget.

J'applaudis à tout ce que vous dites dans votre excellent livre. Si je suis jamais à même de rendre service à la Société, je le ferais avec le plus grand plaisir. Vous savez que je suis de cœur avec vous et toutes vos entreprises.

Bien tout à vous,

Michel Bréal.

\section{Lettre 7}

Cher Monsieur,

Je le [répondre à votre invitation] voudrais bien - pour beaucoup de raisons, mais entre autres pour vous remercier de votre bon souvenir. Mais la marche m'est devenue si difficile que je suis un embarras pour ceux que je vais trouver. Parmi des gymnastes, j'aurais l'air d'une contrepartie ou d'un repoussoir.

Je n'en suis que plus chaudement partisan de la gymnastique. En outre, je suis convaincu que les exercices physiques sont le meilleur antidote et les plus sûrs préservatifs contre la pornographie, dont on vient de parler en termes si justes à la Société des Gens de Lettres. Un gouvernement bien inspiré, un ministre vraiment préoccupé de l'avenir devrait vous aider de tous ses moyens.

Croyez-moi toujours et jusqu'au bout

Votre bien sympathiquant

Michel Bréal 